\title{
Columnista invitado
}

\section{Tecnología y equidad social}

DOI: 10.29236/sistemas.n157a2

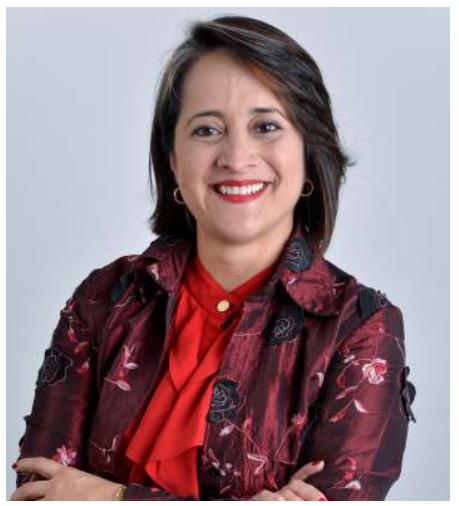

Uno de los conceptos más soñados, políticamente usados y marcados en nuestra comunidad es la equidad social.

\section{Mónica María López S.}

Hablar de equidad social es derribar todos los límites culturales,

Es fácil entender que, cumpliéndose los escenarios de acceso igualitarios a oportunidades y derechos, económicos, sociales y políticos que generan condiciones desiguales para la población y de esta mase garantiza el desarrollo sostenible de una localidad o región. Es nera, establecer condiciones para contar con los mismos derechos y oportunidades. En ese contexto, los escenarios de educación, salud y acceso al trabajo son claves. por esto, que la generación de política pública para facilitar el acceso a espacios de construcción de una sociedad, llevan a la evolución, crecimiento y ética de una zona en la 
que se reconoce de manera respetuosa, el desarrollo de su población.

En los 17 Objetivos de desarrollo sostenible adoptados por la Organización de Naciones Unidas ${ }^{1}$, encontramos otros escenarios en los que se busca erradicar la pobreza, proteger el planeta y asegurar la prosperidad, en el marco de un compromiso compartido de todos los países del planeta para desarrollar políticas y lineamientos de acción, en el logro de dichos objetivos.

\section{Los objetivos de desarrollo sostenible (ODS)}

Para reconocer la importancia del desarrollo y madurez de los escenarios de evolución social, podemos señalar los siguientes: ODS 1 Fin de la pobreza; ODS 2 Hambre cero; ODS 3 Salud y Bienestar; ODS 4 Educación de Calidad; ODS 5 Igualdad de Género; ODS 7 Energía asequible y no contaminante; ODS 11 Ciudades y Comunidades sostenibles y ODS 16 Paz, Justicia e Instituciones sólidas.

Pero tenemos otros ODS clasificados de impacto al medio ambiente, sumados al proceso de equidad social: ODS 6 Agua Limpia y Saneamiento; ODS 13 Acciones por el Clima; ODS 14 Vida submarina y ODS 15 Vida de ecosistemas terrestres.

https://www.un.org/sustainabledevelopment/es/objetivosde-desarrollo-sostenible/
Por otro lado, también algunos de los ODS representan el desarrollo económico:

ODS 8 Trabajo decente y Crecimiento Económico; ODS 9 Industria, Innovación e Infraestructura; ODS 10 Reducción de las desigualdades y ODS 12 Producción y consumo responsable.

Para poder definir el desarrollo de estas acciones y logros de estas metas, figura un último ODS 17 que busca alianzas para el logro de los objetivos.

¿Por qué considero importante que hablemos de equidad social desde los ODS? Porque establecen unos lineamientos globales para la generación de política pública, hasta cambio de mentalidad personal. ¿Y me preguntarán por qué es clave el cambio de mentalidad personal?

Llevamos un poco más de diez años refiriéndonos a la Transformación Digital, dentro de la Cuarta Revolución Digital, entrando a la Quinta y, en medio de esta última, encontramos la influencia y el papel de las tecnologías en el desarrollo, económico, productivo y social de nuestras comunidades y países.

Y es aquí donde podemos enlazar la tecnología con la equidad social.

\section{Las cuatro revoluciones} industriales y la evolución social Las cuatro revoluciones industriales han realizado cambios a partir 
de la inclusión de nuevas tecnologías, que han permeado muchísimos escenarios. Así que reconozcamos características claves en el proceso de desarrollo de cada una de ellas.

\section{Primera Revolución Industrial}

La principal característica de este período fue el inicio del uso de máquinas con el objetivo de aumentar la velocidad con que se desarrollaba el trabajo. Y mediante este método, incrementar también la productividad y las ganancias. Fue una etapa de esplendor económico, con una enorme cantidad de personas operativas y producto de las guerras, que ejercían prácticamente todos los oficios. Se desarrolla y da inicio al proceso de la transmisión hereditaria de las actividades laborales. No existían tantos jefes (en su mayoría era un perfil operativo-técnico). Por otro lado, ante las insatisfacciones laborales surgen los sindicatos.

\section{Segunda Revolución Industrial}

Comienza con la producción a gran escala, la especialización de la mano de obra y con el desarrollo de entidades de formación como centros de aprendizaje. Con una participación de dos terceras partes de la fuerza laboral de mujeres y niños. La falta de condiciones laborales lleva a una alta tasa de mortalidad, surgiendo los derechos laborales y el seguro social con la conciencia de salud ocupacional. Se desarrolla la regulación gubernamental, con actas de responsa- bilidad de los patronos, la creación de leyes compensatorias del trabajo, nuevas estructuras de organización laboral, estableciendo horarios de trabajo y jornadas laborales. Se desarrolló el sector de fábricas y llego el ingreso de las máquinas eléctricas en las industrias.

\section{Tercera Revolución Industrial}

Se caracteriza por la aparición de la microelectrónica y las nuevas tecnologías, la descentralización de la producción, la economía orientada a servicios y la utilización de nuevas formas de energía, especialmente las renovables. Los recursos humanos pasaron a ser mucho más especializados. Esta revolución debía funcionar con un mínimo de mano de obra, en la que se debía lograr una amplia incidencia en todos los aspectos de la vida individual y colectiva, y debía garantizar altos niveles de productividad en los procesos productivos.

Surgió lo smart: smart city (ciudad inteligente), smart grid (distribución inteligente de energía), smart phone, smart TV. Se presentaron formas de transporte más eficientes y menos contaminantes (vehículos eléctricos, híbridos, entre otros). Se inicia la implementación del horario flexible. Surgen las herramientas claves para la nueva gestión productiva, entre ellas la Investigación y Desarrollo, la Implementación de procesos, mayores habilidades de comunicación, continuidad en la formación académica y la automatización. 
Cuarta Revolución Industrial

Vamos andando esta etapa con una capacidad de adaptación constante a la demanda, por parte de la respuesta de las empresas a sus clientes, siendo imprescindible el manejo de la experiencia de cliente, y el servicio se da de una forma más personalizada. Se marca como clave aportar un servicio posventa uno a uno con el cliente. Dada la rapidez de la evolución de productos se requiere diseñar, producir y vender productos en menor tiempo. Se añaden servicios a los productos físicos. Se crean series de producción más cortas y rentables. Es clave aprovechar la información para su análisis, desde múltiples canales en los que es un reto empresarial disponer de la capacidad para analizarla y explotarla en tiempo real. Son claves el desarrollo de mayor motivación y flexibilidad frente a los empleados, la búsqueda de mayor productividad, incluso en menos horas de trabajo (jornada laboral se ajusta a la persona y a la actividad a desarrollar), mayor persuasión, ética laboral, mejor administración de tiempo así como competencias que faciliten la empatía, mayor sensibilidad, la resolución de conflictos, Stress Management, Comunicación (sin importar el idioma o barreras), el desarrollo de escenarios para decisiones descentralizadas, el trabajo colaborativo, los autodidactas (capacitación constante), la adaptación al cambio, cambios en la reglamentación laboral llevando a menos contratos de trabajo fijos, al desarrollo de los nómadas de conocimiento y a la autogestión y autodisciplina en la población en general.

\section{Las áreas de mayor oportunidad} de logro de equidad social

Hemos asociado las diferentes revoluciones industriales al importantísimo aporte de la tecnología frente a los escenarios productivos. Sin embargo, son mucho más los aportes al desarrollo social, en términos de educación, salud, escenarios laborales y es aquí donde, en medio de las condiciones actuales, quiero enfatizar sobre las oportunidades que hoy nos trae la tecnología. Enfoquémonos en tres de las áreas de mayor oportunidad de logro de equidad social.

\section{Educación, tecnología y equidad social (ODS 4)}

La educación no ha cambiado mucho en forma y en fondo, se ha venido adaptando lentamente a las nuevas necesidades por diferentes razones: por reacción lenta, desconocimiento de las nuevas necesidades, desconocimiento de las brechas de conocimiento que faciliten la evolución académica, procesos de aprobación, políticas de gobierno frente a la educación o, sencillamente, el bajo interés de un docente en cambiar el contenido académico. La educación primaria y secundaria viene respondiendo a algunas necesidades básicas, con cambios de contenido, anulación de algunas materias, pero la poca inclusión de nuevas formas de 
educar. La presencialidad sigue marcando el desarrollo de los escenarios de educación, con la inclusión de algunas tecnologías desde la administración y la gestión académica, pero presenta pocos avances en el cambio de mejoras en la formación. Las herramientas tecnologías están en el escenario de la transformación a la educación, solo están presentes en la punta de la pirámide; del total de las entidades, entre públicas y privadas, en las que pensar en contenidos en la nube, el uso de dispositivos electrónicos en lugar de cuadernos y libros y el acceso a herramientas digitales como bibliotecas, laboratorios y simuladores, es de poco acceso a la población que Ilamamos "Nativos Digitales". En el resto de las entidades, la presencia de la tecnología está dada en pocos o nulos escenarios académicos, algunos tableros digitales, proyectores y/o laboratorios dotados de computadores y software especial para educación, sumado a la conectividad; un lujo no necesariamente bien utilizado. $Y$ a pesar de los muchos intentos del Gobierno por llevar computadores y tablets a las entidades de formación, en muchos escenarios sencillamente no se usan por desconocimiento y/o no interés de apropiarlos por parte de los docentes.

En un inicio no tenían contenido académico y aunque en los últimos años ya se generó el contenido, las directivas y los docentes no sienten motivación para generar uso, por temor al daño, además del desinterés por incluirlo en el modelo académico de cada docente.

El confinamiento producido por la COVID-19 llevó a que la tecnología LSM: Learning Manager Sysmet, herramientas de comunicación colaborativa como Teams, Meets, Zoom, Hangout, Skype, inclusive Video por WhatsApp, sean el principal medio para dar acceso a los estudiantes y la oportunidad del docente para cumplir su misión, pero evidenció las carencias alrededor de la educación. Problemáticas claras de abordar: 1. Mayor acceso a conectividad y computadores, tablets o portátiles para el desarrollo de las actividades académicas. 2. Docentes con competencias digitales desarrolladas para garantizar el adecuado uso de las herramientas a la mano. 3. Nuevas metodologías pedagógicas que, de manera remota o virtual, garanticen el desarrollo de las competencias esperadas en el alumno. Y no se suscribe solo a un escenario de evaluación, sino a las nuevas maneras de lograr el desarrollo de conocimientos y capacidades. La usabilidad de tecnologías desarrolladas para la educación remota donde se fortalezcan la inclusión y apropiación de los LMS, Laboratorios y Simuladores Digitales, así como herramientas de gamificación, sistemas de gestión de aprendizaje digital como ClassDojo, EkStep, Moodle, Schoology, sistemas de usabilidad fuera de línea como Kolibry y Rumie. Plataformas para Moocs 
como Canvas, Alison, Coursera, EdX, Future Learn y de contenido de aprendizaje autodirigido como Discovery Education, Duolingo, Code.org, entre otros.

\section{Tecnología y equidad social (ODS 3)}

Durante las cuatro revoluciones industriales, hemos visto cómo ha evolucionado tanto la forma de trabajar como la reglamentación y oportunidad de trabajo. Cada vez se ha requerido mayor especialización, lo que ha permitido la evolución de la formación, creciendo las oportunidades de la mano de obra. Entre más especializado el rol y su responsabilidad, mayor oportunidad de usabilidad de herramientas tecnológicas. Las mismas facilitan su desempeño y el logro de sus resultados.

En los últimos años hemos visto un pánico generalizado, por el boom de las tecnologías que supuestamente van a desaparecer y a remplazar fácilmente cargos y empleados. Eso mismo debió pensar el carretero que en 1886 vio el primer triciclo a motor manejado por la señora Benz. No se qué tantos paros sociales o huelgas se presentaron por parte de estos conductores de carruajes movidos por cuatro patas. Pero lo que sí sé, es que debieron desaprender y reaprender cómo manejar un vehículo Benz o Ford. Y eso mismo vivimos hoy.

Podremos tener nuevos escenarios laborales, que requieren nue- vas competencias técnicas y personales.

Con la COVID-19 y el confinamiento, tanto la educación remota como el teletrabajo y tomaron otro valor. La mayoría de las empresas pudieron seguir su gestión apoyadas en tecnologías. Tuvimos que apropiarnos de aquellas que, durante años, las áreas de tecnología habían tratado de que utilizáramos para facilitar nuestra gestión, CRM, ERP, registros y documentos digitales, mejor administración del correo electrónico, pero también la inclusión de herramientas de comunicación remota como Team o Meets e incluso Zoom, una de las herramientas de mayor crecimiento en usabilidad en el mundo. Sin embargo, perdimos de vista algunas normas de salud y seguridad en el trabajo. Se presentan los jefes desconfiados que tratan de controlar las horas de trabajo en casa. Sencillamente ha sido la forma de sobrevivir como industria. Requiere cambios en la forma de ver el trabajo, confianza y flexibilidad, pero, sobre todo, respeto por la jornada personal.

\section{Salud, tecnología y equidad} social (ODS 3 )

Al igual que la educación, la salud era más presencial que por Telesalud. Sin embargo, ante el escenario de confinamiento se fortaleció la oportunidad de utilizar las tecnologías en atención y consulta. Sigue en evolución, para escenarios de práctica médica, pero con el re- 
conocimiento del profesional tratante dentro de su experiencia y conocimiento, tanto en lo presencial como en lo remoto, lo que requiere reglamentaciones y el reconocimiento económico justo. Sin embargo, las nuevas tecnologías también están presentes. Practicas virtuales, acceso a herramientas de historia clínica digitalizadas por parte del medico tratante, la usabilidad de dispositivos electrónicos para el control de algunos contextos como cardio. Seguimiento a exámenes de manera digital e imagenología. Y sobre todo tecnologías en RV (Realidad Virtual), RA (Realidad Aumentada), Inteligencia Artificial y sensores de loT.

\section{Conclusiones}

Hemos visto la evolución de la tecnología y un sencillo análisis del impacto en la evolución social. La referencia a la transformación digital nos deja en la visibilidad de la tecnología, pero hemos encontrado en los últimos años, que la apropiación $y$, por ende, resultados diferenciales, dependen únicamente del proceso de adopción del ser humano. Pero realmente, ¿podemos reconocer a las tecnologías como ese medio de apertura a nuevos escenarios, con un mayor número de personas?

No hay tales "nativos digitales" (y se que muchos me 'matarán' por esta afirmación). Pero, la pandemia nos mostró que nuestros nativos digitales solo se suscribían a las redes sociales. Ante la necesidad de desarrollar competencias digitales en herramientas académicas, acceso a consultas médicas o laborales, muchos dudaron de la calidad del contenido, solo por no poder tener el control presencial de la situación. Pero como me lo dijo un estudiante en uno de los foros que manejo, "un docente es malo presencial o virtualmente. $\mathrm{O}$ es bueno presencial o virtualmente". Y esto me lleva a confirmar que la tecnología es un medio clave para acceder a más población, y que el contenido solo depende de quien lo desempeñe. Docente, médico o jefeempleado. Pero, definitivamente la tecnología es clave, abriendo puertas de acceso a una mayor población.

Prueba de esto es la democratización de la información a través de internet, para una mayor apertura al conocimiento de un número más amplio de la población. ¿Será que al final la conectividad terminará siendo un derecho o un servicio público? La educación, la salud y el trabajo se han alimentado de esta opción para llegar e un mayor número de personas, descubierta en un escenario de confinamiento. Es claro que necesitamos mayor cubrimiento, acceso y conectividad, además de disponer de dispositivos móviles, pero realmente solo queda una tarea, apropiarnos de ellos, facilitar el logro de los resultados mediando con tecnología dichas labores y entendiendo que lo que necesitamos es un cambio de cultura, cambio en método y flexibi- 
lidad, para lograr hacerlo de una manera diferente.

Cada vez, vemos más presente la tecnología de manera transversal en todos los escenarios productivos, lo que permite que de alguna manera tengamos mayores oportunidades de cambio en nuestros roles y en nuestros resultados.

Definitivamente, podemos llegar a más población con mayores oportunidades y mayores derechos. Pero necesitamos a todos los niveles socioeconómicos no solo exponerlos a estas soluciones a través de escenarios de educación, salud y trabajo entre otros, sino aceptar estos medios como válidos en el logro de oportunidades para todos. Requerimos un cambio cultural, apertura al cambio personal, ética y respeto por las condiciones humanas, para no poner sobre el bien humano, la tecnología; pero, sobre todo, ver las ventajas de la tecnología para el bienestar del ser humano.
¡Bienvenidas la sociedad 5.0 y la Quinta Revolución Industrial!

\section{Referencias}

World Economic Forum (2019). The Global Information Technology Report.

BID (2017). Aprender Mejor. Políticas públicas para el desarrollo de habilidades.

PNUD (2020). ODS en Colombia: Los retos para el 2030.

Soluciones de Aprendizaje a Distancia, página web UNESCO.

Harvard Medical School (2020). Liderazgo de la Transformación Digital en Salud.

UNESCO (2020). Inclusión y Educación.

Alianza TIC (2020). Análisis de Brecha Sectorial TIC en Colombia.

EY (2018). ¿Qué nos depara el futuro?

Mónica María López Sánchez. Psicóloga, especialista en Gerencia de Talento Humano, cursando MBA, con 25 años de experiencia laboral en el desarrollo de procesos que respondan a las necesidades estratégicas empresariales, desde las personas alineadas con los objetivos de negocio, gestión de transformación y transición. Especialista en desarrollo de estrategias de apoyo tecnológico a la educación, investigación e innovación, fortalecidas con tecnología. Ha sido subgerente de Recurso Humano y ejecutiva de Educación y Talento en IBM de Colombia y su subsidiaria. En la actualidad preside la Mesa Sectorial Nacional de Gestión de Tecnología y Talento Digital en Colombia (Ministerio de Trabajo y SENA). Ha sido gerente académica de la Red Nacional de Investigación y Educación (RENATA); directora general de Vinnova SAS y partícipe en la creación y articulación de la Alianza TIC para la medición de la brecha sectorial de talento digital. 